\title{
EFECTO ECOTOXICOLÓGICO AGUDO DEL MERCURIO SOBRE LARVAS DEL "MUY MUY" Emerita analoga (STIMPSON) (DECAPODA: HIPPIDAE) PROCEDENTES DE CUATRO LOCALIDADES DE LIMA
}

José Alberto Iannacone Oliver ${ }^{1}$ y Lorena Alvariño Flores ${ }^{1}$

\begin{abstract}
Resumen
La evaluación de Riesgos Ambientales (ERA) por el mercurio en los ambientes marinos requiere del empleo de especies bioindicadoras. Los organismos biológicos procedentes de localidades con diferentes influencias ambientales presentan respuestas variables en bioensayos ecotoxicológicos. Emerita analoga (Stimpson) es un invertebrado componente del macrozoobentos arenoso marino en la Costa Central del Perú. Se determinó el efecto ecotoxicológico agudo del mercurio $\left(\mathrm{Hg}^{2+}\right)$ sobre larvas de $E$. analoga procedentes de cuatro playas de Lima, Perú: Santa María del Mar, Conchán, El Silencio y Puerto Viejo. Las larvas con menos de $24 \mathrm{~h}$ fueron obtenidas de hembras ovígeras en octubre y noviembre de 1999. Se alimentaron con la microalga Nannochloris sp. y se evaluaron en agua de mar con luz UV. El Diseño en bloques completamente aleatorizado empleado fue de 6 concentraciones $x 4$ repeticiones. Los ensayos ecotoxicológicos a $48 \mathrm{~h}$ y $96 \mathrm{~h}$ de exposición mostraron un valor de Concentración Letal media $\left(\mathrm{CL}_{50}\right)$ promedio de $14,20 \mu \mathrm{g} \mathrm{L}^{-1}$ y $7,48 \mu \mathrm{g} \mathrm{L}^{-1}$, respectivamente. Los parámetros de Concentración de efectos no observables (NOEC) y de Concentración más baja de efectos observables (LOEC) fueron $0,65 \mu \mathrm{g} \mathrm{\textrm {L } ^ { - 1 }}$ y $1,63 \mu \mathrm{g} \mathrm{L}^{-1}$ a $96 \mathrm{~h}$ de exposición, respectivamente. No se encontraron diferencias significativas en los valores de $\mathrm{CL}_{50}$ entre las cuatro localidades evaluadas. El coeficiente de variabilidad (CV) para la $\mathrm{CL}_{50}$ fue aceptable $\left(\mathrm{CV}_{48 \mathrm{~h}}\right.$ $=26,51 \%$ y $\mathrm{CV}_{96 \mathrm{~h}}=40,94 \%$ ). Los resultados son comparados con otras especies de crustáceos acuáticos al nivel mundial. Se analizan las perspectivas del uso de E. analoga como organismo bioindicador de toxicidad.
\end{abstract}

Palabras claves: Crustacea, Emerita, macrozoobentos, mercurio, toxicidad.

\begin{abstract}
Environmental Risk Assessment (ERA) for mercury in marine environment needs the use of bioindicator species. Biological organisms coming from localities with different environmental influences are known to exhibit variable responses in ecotoxicological bioassays. Emerita analoga (Stimpson) is an invertebrate component of marine sand macrozoobenthos in the Peruvian Central coast. The ecotoxicological acute effects of mercury $\left(\mathrm{Hg}^{2+}\right)$ on larvae of $E$. analoga from four beaches of Lima, Peru: Santa María del Mar, Conchan, El Silencio and Puerto Viejo were determined. Less than $24 \mathrm{~h}$ larvae were obtained from ovigerous females in October and November of 1999. They were fed with the Nannochloris sp. microalga and were evaluated using marine water with UV light. A completely randomized block design was employed with 6 concentrations x 4 repetitions. Ecotoxicological assays at $48 \mathrm{~h}$ and $96 \mathrm{~h}$ exposure showed a value of average Lethal medium concentration $\left(\mathrm{LC}_{50}\right)$ of $14,20 \mu \mathrm{g} \mathrm{L}^{-1}$ and $7,48 \mu \mathrm{g} \mathrm{L}^{-1}$, respectively. Non Observed Effect Concentration (NOEC) and Lowest Observed Effect Concentration (LOEC) were $0,65 \mu \mathrm{g} \mathrm{L} \mathrm{L}^{-1}$ and $1,63 \mu \mathrm{g} \mathrm{L}^{-1}$ at $96 \mathrm{~h}$ exposure, respectively. Non significant differences in values of $\mathrm{LC}_{50}$ among the four localities evaluated were observed. Variability coefficient (VC) of $\mathrm{LC}_{50}$ was acceptable $\left(\mathrm{VC}_{48 \mathrm{~h}}=26.51 \%\right.$ and $\left.\mathrm{VC}_{96 \mathrm{~h}}=40,94 \%\right)$. The results were compared with other species of aquatic crustaceans at the global level. Perspectives of use of E. analoga as a toxicity bioindicador organism were analyzed.
\end{abstract}

Key word: Crustacea, Emerita, macrozoobenthos, mercury, toxicity.

\section{Introducción}

Emerita analoga (Stimpson 1857) "Muy Muy" es un crustáceo macrozoobentónico característico del intermareal e infralitoral de las playas de arena de las Costas del Pacífico Sur de América, a la vez que es un eslabón importante en las tramas tróficas de este sistema (Oliva et al., 1992; Alvitrés et al., 1999; Contreras et al., 2000). Pese a su gran importancia pocos son los estudios tendientes a conocer aspectos de su ecología (Paul, 1976; Iannacone et al., 1996;

\footnotetext{
${ }^{1}$ Laboratorio de Ecofisiología Animal. Facultad de Ciencias Naturales. Universidad Nacional Federico Villarreal (FCCNM-UNFV). Calle San Marcos 383, Lima 21, Perú. Correo electrónico: joseiannacone@hotmail.com / joselorena@terra.com
} 
Homola \& Chang, 1997; Faulkes \& Paul, 1998; Paul \& Bruner, 1999).

E. analoga ha sido empleada como especie bioindicadora de importancia ecotoxicológica para el monitoreo de algunos metales pesados (Hernández et al., 2000; Valdovinos \& Zúñiga, 2002), de efluentes pesqueros y de ficotoxinas (Bretz et al., 2002; Ferdin et al., 2002; Powell et al., 2002) en el ecosistema marino.

Los organismos biológicos procedentes de localidades con diferentes características ambientales podrían presentan diferentes respuestas en bioensayos ecotoxicológicos (Forbes, 1998; Repetto et al., 2000; Alayo \& Iannacone, 2002).

La contaminación por mercurio produce efectos deletéreos en diferentes componentes de la biota acuática (Pritchard, 1993; Rainbow, 1997; Iannacone \& Alvariño, 1999). Se han hecho evaluaciones del efecto del mercurio $\left(\mathrm{Hg}^{2+}\right)$ y del metilmercurio $\left(\mathrm{MeHg}^{2+}\right)$ en el ambiente acuático (Baker et al., 1985; Pritchard, 1993; Andres et al., 2002).

Según la Ley General de Aguas en el Perú, los límites máximos permisibles para el $\mathrm{Hg}^{2+}$ en aguas de Clase V para el cultivo de moluscos es de $0,1 \mu \mathrm{g} \mathrm{L}^{-1}, \mathrm{y}$ según la Agencia de Protección ambiental de los Estados Unidos (USEPA), es de $2 \mu \mathrm{g} \mathrm{L}^{-1}$ para la protección de los ambientes acuáticos naturales.

En el Perú, se han evaluado del ambiente litoral marino a las siguientes tres especies: Tetrapigus niger (Molina) "erizo negro", Arbacia spatuligera (Valenciennes) “erizo marrón” y Perumytilus purpuratus (Lamarck) (Iannacone et al., 1999; 2001).

Muchas formas larvales y postlarvales de crustáceos marinos han sido empleadas para evaluar la ecotoxicidad de metales pesados, por constituir los estadios más sensibles de su ciclo de vida (MarinoBalsa et al., 2000).

El objetivo fue determinar el efecto ecotoxicológico agudo del mercurio $\left(\mathrm{Hg}^{2+}\right)$ sobre zoeas de E. analoga procedentes de cuatro Playas de la localidad de Lima: El silencio, Santa María del Mar, Conchán y Puerto Viejo.

\section{Materiales y métodos}

\section{Emerita analoga}

Se procedió a la colección, identificación y cría estandarizada de $E$. analoga. Cincuenta hembras de $E$. analoga con presencia de masas ovígeras fueron colectadas de cada una de las cuatro playas arenosas: El Silencio, Santa María del Mar, Conchán y Puerto Viejo de Lima, Perú durante octubre y noviembre en 1999. Los ensayos ecotoxicológicos con E. analoga se iniciaron con larvas o "zoeas" de primer estadio dentro de $24 \mathrm{~h}$ de haber eclosionado de las masas ovígeras procedentes de cada una de las cuatro playas. Diez larvas de primer estadio se distribuyeron al azar en cada concentración nominal de $\mathrm{Hg}^{2+}$ evaluada en cada una de las cuatro repeticiones del ensayo experimental. Las larvas se consideraron muertas si no fueron capaces de moverse coordinada y normalmente cuando fueron pinchadas ligeramente con un alfiler luego de $48 \mathrm{~h}$ y $96 \mathrm{~h}$ de exposición. Se emplearon un total de 240 larvas por ensayo. Los bioensayos semiestáticos con renovación de la solución a $48 \mathrm{~h}$ de exposición fueron repetidos 2 veces en envases plásticos de $0,5 \mathrm{~L}$. La temperatura se mantuvo en una incubadora fluctuante entre $19{ }^{\circ} \mathrm{C} \pm 3{ }^{\circ} \mathrm{C}$.

\section{Mercurio y Físico-química del agua}

La solución madre para el ión tóxico de mercurio fue preparada de la sal metálica a base del cloruro de mercurio $\left(\mathrm{HgCl}_{2}\right)$ (Iannacone \& Alvariño, 1999) a una concentración de $100 \quad \mathrm{mg} \quad \mathrm{L}^{-1}$. Todas las concentraciones se registraron en concentraciones nominales de ión de mercurio. Los matraces aforados previamente fueron lavados con ácido nítrico al $10 \%$ y luego enjuagados en agua destilada y mantenidos a 4 ${ }^{\circ} \mathrm{C}$. (Iannacone et al., 2000). Los datos de oxígeno disuelto se midieron mediante el método de Winkler (APHA, 1995). En el ensayo con $\mathrm{Hg}^{2+}$ se emplearon las siguientes cinco concentraciones $0,65 \mu \mathrm{g} \mathrm{L}^{-1}, 1,63 \mu \mathrm{g}$ $\mathrm{L}^{-1}, 4,09 \mu \mathrm{g} \mathrm{L} \mathrm{L}^{-1}, 10,24 \mu \mathrm{g} \mathrm{L^{-1 }}$ y $25,60 \mu \mathrm{g} \mathrm{L} \mathrm{L}^{-1}$ (Iannacone \& Alvariño, 2002). Para el $\mathrm{Hg}^{2+}$ las cinco concentraciones siguieron un factor de incremento en 2,5 .

\section{Diseño experimental y Análisis de datos}

Se determinaron la Concentración Letal media $\left(\mathrm{CL}_{50}\right)$ promedio, su desviación estándar y su Coeficiente de Variación ( $\mathrm{CV} \%$ ) para todos los bioensayos con E. analoga expuestos al mercurio a 48 y $96 \mathrm{~h}$ de exposición para las cuatro localidades evaluadas. Las diferencias entre las concentraciones y entre las repeticiones se evaluaron a través de un Análisis de Varianza (ANDEVA) de dos vías, previa transformación de los datos a raíz cuadrada del arcoseno, con el fin de ajustar los datos a la distribución normal (Zar, 1996). En el caso de existir diferencias significativas entre las concentraciones y entre las repeticiones se realizó una Prueba de Significación DVS (Diferencia Verdaderamente Significativa) de Tukey (Daniel, 1993).

Las $\mathrm{CL}_{50} \mathrm{~S}$ se calcularon usando un programa computarizado de la EPA versión 1,5 (USEPA, 1993). Estos valores fueron obtenidos a partir del análisis de regresión lineal entre el logaritmo de la concentración de dilución de las muestras de agua (en porcentaje) y la mortalidad Probit (porcentaje transformado). Los porcentajes de mortalidad de las repeticiones para cada concentración fueron sumados. El modelo de regresión lineal fue verificado usando el estadístico $\chi^{2}$ (Martín \& Holdich, 1986). Se determinó además el NOEC y el LOEC a 48 h y 96 h de exposición.

\section{Resultados y discusión}

Las variaciones de tres parámetros ecotoxicológicos y el CV respectivo para el crustáceo de E. analoga expuesto al mercurio son señalados en la Tabla 1. 
Tabla 1. Valores de $\mathrm{CL}_{50}$, NOEC y LOEC del mercurio sobre larvas de Emerita analoga procedentes de cuatro playas de Lima, Perú.

\begin{tabular}{lccccccc}
\hline $\begin{array}{l}\text { Parámetros } \\
\left(\text { ug L }^{-1}\right)\end{array}$ & El SilencioSanta MaríaConchánPuerto Viejo & $\begin{array}{c}\text { Promedio } \\
\pm \mathrm{DE}\end{array}$ & $\begin{array}{c}\text { Coeficiente } \\
\text { de variación }\end{array}$ \\
\hline $\mathrm{CL}_{50} 48 \mathrm{~h}$ & 10,60 & 10,96 & 12,92 & 18,18 & $13,16 \pm 3,49$ & 26,51 \\
$\mathrm{CL}_{50} 96 \mathrm{~h}$ & 3,16 & 8,69 & 5,40 & 8,37 & $6,40 \pm 2,62$ & 40,94 \\
NOEC $48 \mathrm{~h}$ & 1,63 & 4,09 & 0,65 & 4,09 & $2,61 \pm 1,74$ & 66,66 \\
NOEC 96 h & $<0,65$ & 1,63 & 0,65 & 0,65 & $0,89 \pm 0,49$ & 55,05 \\
LOEC 48 h & 4,09 & 10,24 & 1,63 & 10,24 & $6,55 \pm 4,37$ & 66,71 \\
LOEC 96 h & 0,65 & 4,09 & 1,63 & 1,63 & $2,00 \pm 1,46$ & 73
\end{tabular}

NOEC $=$ Concentración de efectos no observables.

LOEC $=$ Concentración más baja de efectos observables.

$\mathrm{DE}=$ Desviación estándar.

Los porcentajes de mortalidad de las larvas de $E$. analoga aumentaron con cada una de las concentraciones crecientes de $\mathrm{Hg}^{2+}$ a 48 y $96 \mathrm{~h}$ de exposición (Tablas 2 y 3 ).

Tabla 2. Porcentaje de Mortalidad de E. analoga expuestas al mercurio a $48 \mathrm{~h}$.

\begin{tabular}{|c|c|c|c|c|c|c|}
\hline & \multicolumn{7}{|c|}{ (\% mortalidad) } & \\
\hline $\mathrm{Mg} \mathrm{L}^{-1}$ & 1 & 2 & 3 & 4 & Promedio $\pm \mathrm{DE}$ & Sig. \\
\hline Control & $0 \mathrm{a}$ & $0 \mathrm{a}$ & $0 \mathrm{a}$ & $0 \mathrm{a}$ & $0 \pm 0$ & $\mathrm{a}$ \\
0,65 & $0 \mathrm{a}$ & $0 \mathrm{a}$ & $5 \mathrm{a}$ & $5 \mathrm{a}$ & $2,5 \pm 2,88$ & $\mathrm{a}$ \\
1,63 & $1,13 \mathrm{a}$ & $0 \mathrm{a}$ & $13,33 \mathrm{~b}$ & $5 \mathrm{a}$ & $4,86 \pm 6,03$ & $\mathrm{ab}$ \\
4,09 & $8,88 \mathrm{~b}$ & $2,50 \mathrm{a}$ & $15 \mathrm{~b}$ & $10 \mathrm{ab}$ & $9,09 \pm 5,13$ & $\mathrm{~b}$ \\
10,24 & $50,60 \mathrm{c}$ & $37,50 \mathrm{~b}$ & $26,19 \mathrm{c}$ & $17,50 \mathrm{~b}$ & $32,94 \pm 14,33$ & $\mathrm{c}$ \\
25,60 & $87,31 \mathrm{~d}$ & $100 \mathrm{c}$ & $82,50 \mathrm{~d}$ & $77,50 \mathrm{c}$ & $86,82 \pm 9,65$ & $\mathrm{~d}$ \\
\hline
\end{tabular}

1 =El Silencio; 2 = Santa María; 3 = Conchán; 4 = Puerto Viejo. $\mathrm{DE}=$ Desviación estándar; Sig. $=$ Significancia.

Letras iguales en una misma columna indican que los promedios son estadísticamente iguales.

La secuencia ecotoxicológica en términos de $\mathrm{CL}_{50}$ de mayor a menor toxicidad fue: Daphnia obtusa $>$ Daphnia pulex $>$ Daphnia cucullata $>$ Daphnia obtusa $>$ Pagurus longicarpus $>$ Daphnia magna $>$ Emerita analoga $(96 \mathrm{~h})>$ Daphnia magna $>$ Emerita analoga $(48 \mathrm{~h})>$ Corophium insidiosum $>$ Elasmopus bampo $>$ Homarus gammarus $>$ Streptocephalus proboscideus $>$ Thamnocephalus platyurus > Maja squinado > Palaemon serratus (Tabla 4). E. analoga ocupa la posición $7^{\text {ma }}$ entre 16 bioensayos ecotoxicológicos empleando diferentes especies carcinológicas, componentes de la biota animal acuática (Tabla 4).

McKinney \& Rogers (1992) han señalado al mercurio como uno de los metales de mayor interés por la USEPA (Agencia de Protección Ambiental de los Estados Unidos), por lo que requiere colaboración entre toxicólogos y químicos para coordinar investigaciones en la química de la especiación de metales y en los efectos toxicológicos de la especiación de metales (Wright, 1995).

Alayo \& Iannacone (2002) presentan una evaluación comparativa global entre E. analoga,
Brachionus plicatilis Müller 1786 (Rotifera) y Artemia salina (L.) (Crustacea) para su eventual empleo en bioensayos ecotoxicológicos. Entre los criterios usados para validar un bioensayo tenemos: "facilidad de uso" que incluye rapidez del bioensayo, simplicidad en la manipulación, disponibilidad del material biológico, poco volumen de muestra y facilidad en la observación y conteo de los organismos afectados (muertos o inmóviles). Los decápodos como E. analoga permiten realizar pruebas de fácil lectura, pero con manipulación simple. La simplicidad de manipulación y lectura dependen esencialmente del tamaño de los organismos; a mayor tamaño más simple será el método. Por ello, Brachionus al poseer menor tamaño que Emerita, presenta mayor dificultad en su manejo. E. analoga es una especie con alta disponibilidad en el Perú, como material biológico para bioensayos. El criterio de "costo" incluye lo invertido en materiales y los gastos del trabajo, tanto en el cultivo como en la prueba de toxicidad. Los bioensayos con Brachionus son menos costosos que utilizar Emerita o Artemia. La "concordancia ecológica" en el ambiente marino favorece principalmente a E. analoga por su importancia en las redes tróficas.

Tabla 3. Porcentaje de Mortalidad de E. analoga expuestas al mercurio a $96 \mathrm{~h}$.

\begin{tabular}{|c|c|c|c|c|c|c|}
\hline & \multicolumn{7}{|c|}{$(\%$ mortalidad $)$} & \\
\hline$\mu \mathrm{g} \mathrm{L}^{-1}$ & 1 & 2 & 3 & 4 & Promedio $\pm \mathrm{DE}$ & Sig. \\
\hline control & $0 \mathrm{a}$ & $0 \mathrm{a}$ & $0 \mathrm{a}$ & $0 \mathrm{a}$ & $0 \pm 0$ & $\mathrm{a}$ \\
\hline 0,65 & $21,22 \mathrm{~b}$ & $2,29 \mathrm{a}$ & $5 \mathrm{a}$ & $3,87 \mathrm{a}$ & $8,09 \pm 8,82$ & $\mathrm{~b}$ \\
\hline 1,63 & $39,40 \mathrm{c}$ & $1,30 \mathrm{a}$ & $33,33 \mathrm{~b}$ & $10,73 \mathrm{~b}$ & $28,69 \pm 12,45$ & $\mathrm{c}$ \\
\hline 4,09 & $33,34 \mathrm{c}$ & $11,92 \mathrm{~b}$ & $25,64 \mathrm{~b}$ & $45 \mathrm{c}$ & $28,97 \pm 13,87$ & $\mathrm{c}$ \\
\hline 10,24 & $84,85 \mathrm{~d}$ & $57,38 \mathrm{c}$ & $52,38 \mathrm{c}$ & $72,23 \mathrm{~d}$ & $66,71 \pm 14,74$ & $\mathrm{~d}$ \\
\hline 25,60 & $100 \mathrm{e}$ & $100 \mathrm{~d}$ & $100 \mathrm{~d}$ & $100 \mathrm{e}$ & $100 \pm 0$ & $\mathrm{e}$ \\
\hline $1=$ El Silencio; $2=$ Santa María; $3=$ Conchán; $4=$ Puerto Viejo. \\
DE = Desviación estándar; Sig. = Significancia. & \\
Letras iguales en una misma columna indican que los promedios son \\
estadísticamente iguales.
\end{tabular}

Muchas especies propuestas como herramientas para bioensayos ecotoxicológicos, requieren como requisitos para uso no solo que sean sensibles, sino que sean ensayos reproducibles con poca variabilidad en su respuesta. En el caso de E. analoga al provenir de cuatro localidades diferentes de la costa central de Lima, Perú, no presentó diferencias significativas entre los valores de $\mathrm{CL}_{50}$, NOEC y LOEC para el $\mathrm{Hg}^{2+}$ a 48 y 96 h de exposición (Tabla 1). Finalmente, $E$. analoga en su fase larvaria en el ciclo de vida de esta especie es apropiada para su empleo en bioensayos ecotoxicológicos para evaluar el metal $\mathrm{Hg}^{2+}$ en el ambiente acuáticos marino. 
Tabla 4. Valores comparativos de $\mathrm{CL}_{50}$ con especies de crustáceos marinos y dulceacuícolas expuestos al mercurio.

\begin{tabular}{|c|c|c|c|c|c|}
\hline Especie de Crustáceos & $\mathrm{CL}_{50} \mu \mathrm{g} \mathrm{L}^{-1}$ & $\begin{array}{c}\text { Tiempo de } \\
\text { exposición(h) }\end{array}$ & Estadio & Ambiente & Referencia \\
\hline Daphnia obtusa & 2 & 48 & Neonato & $\mathrm{D}$ & Rossini \& Romo (1996) \\
\hline Daphnia pulex & 2,2 & 48 & Neonato & $\mathrm{D}$ & Rossini \& Romo (1996) \\
\hline Daphnia cucullata & 2,4 & 48 & Neonato & $\mathrm{D}$ & Rossini \& Romo (1996) \\
\hline Daphnia obtusa & 4 & 24 & Neonato & $\mathrm{D}$ & Rossini \& Romo (1996) \\
\hline Pagurus longicarpus & 4 & 168 & Adultos & M & Eisler \& Hennekey (1977) \\
\hline Daphnia magna & $1,5-3,2$ & 48 & Neonato & $\mathrm{D}$ & Rossini \& Romo (1996) \\
\hline Emerita analoga & 6,40 & 96 & Primero & M & Original \\
\hline Daphnia magna & 9,2 & 48 & Neonato & $\mathrm{D}$ & Brkovic-Popovic (1990) \\
\hline Emerita analoga & 13,16 & 48 & Primero & M & Original \\
\hline Corophium insidiosum & 20 & 96 & Adultos & M & Reish (1993) \\
\hline Elasmopus bampo & 20 & 96 & Adultos & M & Reish (1993) \\
\hline Homarus gammarus & 48 & 48 & Primero & M & Marino-Balsa et al. (2000) \\
\hline Streptocephalus proboscideus & 60 & 24 & Nauplio & $\mathrm{D}$ & Centeno et al. (1995) \\
\hline Thamnocephalus platyurus & 60 & 24 & Nauplio & $\mathrm{D}$ & Centeno et al. (1995) \\
\hline Maja squinado & 72 & 72 & Primero & $\mathrm{M}$ & Marino-Balsa et al. (2000) \\
\hline Palaemon serratus & 74 & 72 & Primero & $\mathrm{M}$ & Marino-Balsa et al. (2000) \\
\hline
\end{tabular}

$\mathrm{M}=$ marino; $\mathrm{D}=$ dulceacuícola.

\section{Literatura citada}

Alayo M. \& Iannacone J. 2002. Ensayos ecotoxicológicos con petróleo crudo, Diesel 2 y Diesel 6 con dos subespecies de Brachionus plicatilis Müller 1786 (Rotifera: Monogononta). Gayana. 66: 45-58.

Alvitres V., Chanamé J., Fupuy J., Chambergo A. \& Cortez M. 1999. Cambios en la prevalencia de los helmintos parásitos de Emerita analoga por efecto de "El Niño 1997-97". Rev. per. Biol. Vol. Extraordinario.: 69-76.

Andres S., Laporte J.M. \& Mason R.P. 2002. Mercury accumulation and flux across the gills and the intestine of the blue crab (Callinectes sapidus). Aquat. Toxicol. 56:303-20.

APHA (AMERICAN PUBLIC HEALTH ASSOCIATION), AWWA (AMERICAN WATER WORKS ASSOCIATION), WPCF (WATER POLLUTION CONTROL FEDERATION). 1995. Standard methods for examination of water and wastewater. $19^{\text {th }}$. Ed. American Health Association. Washington, D.C.

Baker R., Lavie B. \& Nevo E. 1985. Natural selection for resistance to mercury pollution. Experientia. 41: 697-699.

Brkovic-Popovic I. 1990. Effect of mercury on the survival of Daphnia magna. Wat. Sci. Tech. 22: 241-246.

Bretz C.K., Manouki T.J. \& Kvitek R.G. 2002. Emerita analoga (Stimpson) as an indicator species for paralytic shellfish poisoning toxicity along the California Coast. Toxicon. 40: 11891196.
Centeno M.D.F., Perssone G. \& Goyvaerts M.P. 1995. Cyst-based toxicity test. IX. The potential of Thamnocephalus platyurus as test species in comparison with Streptocephalus proboscideus (Crustacea: Brachipoda: Anostraca). Environm. Toxicol. Water Qual. 10: 275-282.

Contreras H., Jaramillo E. \& Quijón P. 2000. Natural history of Emerita analoga (Stimpson) (Anomura, Hippidae) in a sandy beach of northern Chile. Rev. chil. Hist. nat. 73: 705-715.

Daniel W.W. 1993. Bioestadística. Tercera Edición. Editorial Limusa (México).

Faulkes Z. \& Paul D.H. 1998. Digging in sand crabs: coordination of joints in individuals legs. J. Exp. Biol. 201: 2139-2149.

Ferdin M.E., Kvitek R.G., Bretz C.K., Powell C.L., Doucette G.J., Lefebvre K.A. \& Coale S. 2002. Emerita analoga (Stimpson) - possible new indicator species for the phycotoxin domoic acid in California coastal waters. Toxicon. 40: 1259-1265.

Forbes V.E. 1998. Sources and implications of variability in sensitivity to chemicals for ecotoxicological risk assessment. Arch. Toxicol. Suppl. 20: 407-418.

Hernández C., Yánez R. \& Rudolph A. 2000. Toxicity response of Emerita analoga (Stimpson, 1857) collected from beaches of South Central Chile. Bull. Environ. Contam. Toxicol. 65: 567-572.

Homola E. \& Chang E.S. 1997. Distribution and regulation of esterases that hydrolyze methyl farnesoate in Homarus americanus and other crustaceans. Gen. Comp. Endocrinol. 106: 62-72. 
Iannacone J. \& Alvariño L. 1999. Acute ecotoxicity of heavy metals using juveniles of freshwater snail Physa venustula (Gould, 1847) (Mollusca). Gayana. 63: 101-110.

Iannacone J. \& Alvariño L. 2002. Efecto del detergente doméstico alquil aril sulfonato de sodio lineal (LAS) sobre la mortalidad de tres caracoles dulceacuícolas en el Perú. Ecol. Apl. 1: 81-87.

Iannacone J., Alvariño L., Dionisio M. \& Sánchez J. 1999. Efecto del $\mathrm{Cu}^{2+}$ en la embrionación del erizo negro Tetrapigus niger (Molina) y en la fertilización del erizo marrón, Arbacia spatuligera (Valenciennes) (Echinodermatha). Bol. Soc. Quim. Peru. 65: 183-190.

Iannacone J., Dale W. \& Alvariño L. 2000. Monitoreo ecotoxicológico del río Rímac (Lima-Perú) empleando a Chironomus calligraphus Goeldi (Diptera: Chironomidae). Rev. Chil. Ent. 27: 2534.

Iannacone J., García J., Vela H., Ticona C., Torres E., Quinte G. \& Vidarte K. 2001. Toxicidad y bioacumulación de plomo en Perumytilus purpuratus (Lamarck, 1819) "chorito" (Bivalvia). Bol. Soc. Quim. Peru. 67: 89-98.

Iannacone J., Villaseca A. \& Mari C. 1996. Algunos aspectos ecológicos de la fauna parasitaria (metazoa) de Callianassa garthi "marucha" y Emerita analoga "muy muy" en la costa central del Perú. Congreso Nacional de Ecología. 25-28 de marzo 1996. Lima, Perú.

Lanston W.J. 1990. Toxic effects of metals and the incidence of metal pollution in marine ecosystems. pp. 101- 119. In: Heavy metals in the marine environment (Furness, R.W. \& Rainbow, P. S., eds.). CRC Press, Inc. Boca Raton, Florida, USA.

Marino-Balsa J.C., Poza. E., Vazquez. E. \& Beiras, R. 2000. Comparative toxicity of dissolved metals to early larval stages of Palaemon serratus, Maja squinado, and Homarus gammarus (Crustacea: Decapoda). Arch. Environ. Contam. Toxicol. 39: 345-351.

Martin T.R. \& Holdich D.M. 1986. The acute lethal toxicity of heavy metals to peracarid crustaceans (with particular reference to freshwater asellids and gammarids). Water Res. 20: 1137-1147.

McKinney J. \& Rogers R. 1992. Metal bioavailability: EPA workshop identified research needs. Environ. Sci. Technol. 26: 1298-1299.
Oliva M.E., Luque J.L. \& Cevallos A. 1992. Parásitos de Emerita analoga (Stimpson) (Crustacea): Implicancias ecológicas. Bol. Lima (Perú). 79: 7780.

Paul D.H. 1976. Role of proprioceptive feedback from nonspiking mechanosensory cells in the sand crab, Emerita analoga. J. Exp. Biol. 65: 243-258.

Paul D.H. \& Bruner J. 1999. Receptor potentials and electrical properties of nonspiking strech-receptive neurons in the sand crab Emerita analoga (Anomura : Hippidae). J. Neurophysiol. 81: 24932500 .

Powell C.L., Ferdin M.E., Busman M., Kvitek R.G. \& Doucette G.J. 2002. Development of a protocol for determination of domoic acid in the sand crab (Emerita analoga): a possible new indicator species. Toxicon 40: 485-492.

Pritchard J.B. 1993. Aquatic Toxicology: past, present, and prospects. Environ. Health Perspectives. 100: 249-257.

Rainbow P.S. 1997. Trace metals accumulation in marine invertebrates: marine biology or marine chemistry. J. mar. biol. Ass. U.K. 77: 195-210.

Reish D.J. 1993. Effect of metals and organic compounds on survival and bioaccumulation in two species of marine gammaridean amphipod, together with a summary of toxicological research on this group. Jour. Nat. History. 27: 781-794.

Repetto G., Peso A. \& Repetto M. 2000. Alternative ecotoxicological methods for the evaluation, control and monitoring for environmental pollutants. Ecotoxicol. Environm. Res. 3: 47-51.

Rossino G.B. \& Ronco A.E. 1996. Acute toxicity bioassay using Daphnia obtusa as a test organism. Environm. Toxicol. Water Qual. 11: 255-258.

USEPA. 1993. Probit analysis program. Version 1,5. U.S. Environmental Protection Agency. Washington D.C. U.S.A.

Valdovinos C. \& Zuñiga M. 2002. Copper acute toxicity tests with the sand crab Emerita analoga (Decapoda: Hippidae): a biomonitor of heavy metal pollution in Chilean coastal seawater?. Bull. Environ. Contam. Toxicol. 69: 393-400.

Wright D.A. 1995. Trace metals and major ion interactions in aquatic animals. Mar. Poll. Bull. 34: 8-17.

Zar J.H. 1996. Bioestatistical Analysis. $3^{\text {th }}$ Ed. Prentice -Hall. Inc. Upper Saddle River, Jew Jersey. 